\title{
HEAO-1 DIFFUSE SOFT X-RAY SKY MAPS
}

John A. Nousek, Gordon P. Garmire, and George Weaver

Department of Astronomy, The Pennsylvania State University

\section{ABSTRACT}

Maps of the diffuse soft X-ray background intensity are presented, spanning four energy intervals. The lowest energy interval (0.18-0.56 keV) is dominated by local emission, while the next two intervals $(0.56-1.0 \mathrm{keV}$ and 1.0-1.4 keV) reveal more distant and more sharply defined structures. Enlarged maps of several of these structures are presented, including the North Polar Spur, the Galactic Center region and the Eridanus Loop.

\section{INTRODUCTION}

The HEAO-1 Satellite A-2 LED (Low Energy Detectors) experiment surveyed the sky at soft X-ray energies using thin window proportional counters. Despite a premature end of the survey due to depletion of the counting gas more than $95 \%$ of the sky was observed during its nine months of operation.

Although many point sources of soft X-rays were studied with the LED (cf. the source catalog, Nugent et al 1983), its combination of respectable collecting area ( $400 \mathrm{~cm}^{2}$ ), moderate solid angle ( $2.2 \mathrm{msr}$ ), long integration time ( 100 hours) and broad sky coverage make the LED data an important source for study of the diffuse soft X-ray background.

A serious problem with all efforts to study the diffuse sky X-ray background with proportional counters is the presence of low energy particles able to penetrate the thin window and mimic X-ray events in the detector. Evidence of such contamination can be seen in the LED data, despite the presence of magnetic brooms designed to prevent charged particles from entering the detector. The contamination appears in our data as long streaks at constant ecliptic longltude. Fortunately most of the charged particle background can be rejected by employing data taken from the central detector volume. Charged particles with energies low enough to mimic soft $\mathrm{X}$-rays are stopped before they enter this volume.

Such residual non-X-ray background as remains in the current maps is not easily explained by charged particles. In some cases every indication points to a true X-ray origin of these events. A speculation, unpalatable because of the high electron flux required, is that some of these noncelestial X-rays are produced by fluorescence of the residual atmosphere near the satellite. 
Regardless of the nature of the contamination, the residual in these maps is no more than $20 \%$ of the diffuse sky intensity, as infered from the fluctuations.

MAPS

The LED data were collected in one detector having two separate mechanically collimated fields of view. The response of each is approximately triangular in orthogonal directions, being $1.55^{\circ} \times 2.95^{\circ}$ FWHM for one (L), and $2.80^{\circ} \times 2.55^{\circ}$ in the other (R). The collecting areas are $176.5 \mathrm{~cm}^{2}$ for $\mathrm{L}$, and $205 \mathrm{~cm}^{2}$ for $\mathrm{R}$.

The collecting volume is surrounded on front, back and sides by active veto volumes, rejecting charged particles. Data have also been excluded from geographical regions of high background.

The data are grouped into pulse helght bins prior to display. The response of the four intervals is shown in Fig. 1. Note that the absorption due to the front layer causes the sensitivity of the intervals to be less at low energy than the nominal pulse height labelling would imply. Thus the $0.56-1.0 \mathrm{keV}$ band, for example, has half response points at 0.75 and $1.2 \mathrm{keV}$.

\section{DISCUSSION}

The higher resolution and longer exposure of the HEAO-I LED provide a better glimpse of the intricate structure of the soft X-ray background. At $1 / 4 \mathrm{keV}$ the features are largely diffuse. A reasonable explanation for the origin of this radiation is in local emission from a hot plasma. Near $1 \mathrm{keV}$ the features are better defined, hinting at a more distant origin.

The true test for the origin of this radiation must come from detailed comparison, at comparable resolution, of the X-ray intensity with other aspects of the interstellar medium, for example $21-\mathrm{cm}$ radio data, interstellar extinction, and ultraviolet absorption.

\section{ACKNOWLEDGEMENT}

David Burrows provided Fig. 1. Ryland Truax assisted with the image processing computer. This work was sponsored by NAS contract NAS 5-52049.

\section{REFERENCE}

Nugent, J.J. et a1. 1983, Ap.J. Supp1. 미, 1. 
Efficiency of each pulse height interval to a mono-energetic flux as a function of energy. A-0.18-0.56 keV band. B-0.56-1.0 keV band. $\mathrm{C}-1.0-1.4 \mathrm{keV}$ band. D-1.4-2.8 keV band.

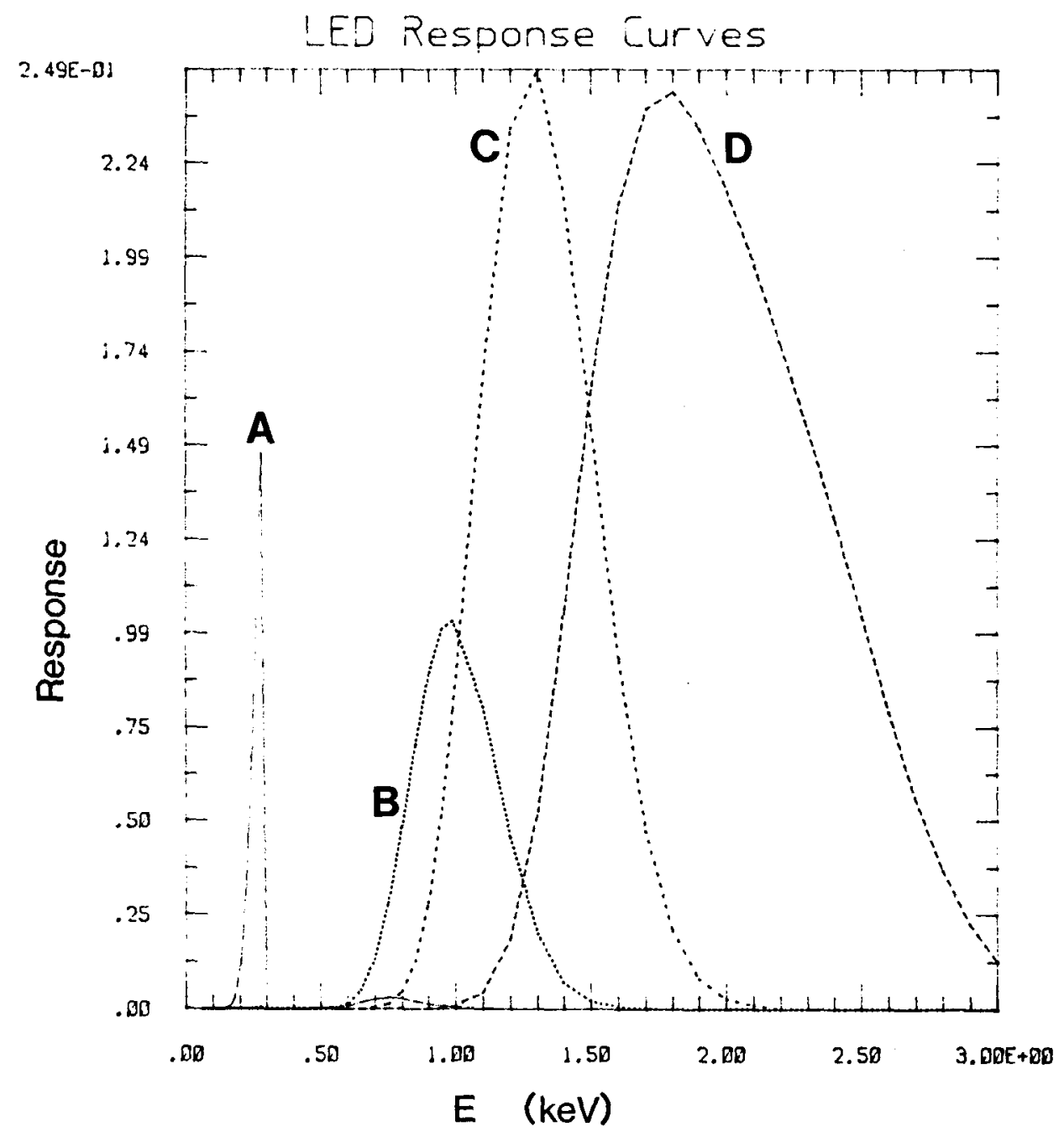

